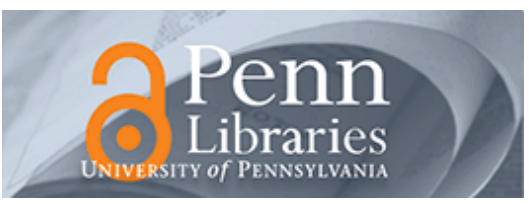

University of Pennsylvania ScholarlyCommons

June 1992

\title{
The Fast Multipole Method (FMM) for Electromagnetic Scattering Problems
}

\author{
Nader Engheta \\ University of Pennsylvania, engheta@ee.upenn.edu \\ William D. Murphy \\ Rockwell International \\ Vladimir Rokhlin \\ Yale University \\ Marius S. Vassiliou \\ Rockwell International Science Center
}

Follow this and additional works at: https://repository.upenn.edu/ese_papers

\section{Recommended Citation}

Nader Engheta, William D. Murphy, Vladimir Rokhlin, and Marius S. Vassiliou, "The Fast Multipole Method (FMM) for Electromagnetic Scattering Problems", . June 1992.

Copyright 1992 IEEE. Reprinted from IEEE Transactions on Antennas and Propagation, Volume 40, Issue 6, June 1992, pages 634-641.

This material is posted here with permission of the IEEE. Such permission of the IEEE does not in any way imply IEEE endorsement of any of the University of Pennsylvania's products or services. Internal or personal use of this material is permitted. However, permission to reprint/republish this material for advertising or promotional purposes or for creating new collective works for resale or redistribution must be obtained from the IEEE by writing to pubs-permissions@ieee.org. By choosing to view this document, you agree to all provisions of the copyright laws protecting it.

This paper is posted at ScholarlyCommons. https://repository.upenn.edu/ese_papers/193

For more information, please contact repository@pobox.upenn.edu. 


\title{
The Fast Multipole Method (FMM) for Electromagnetic Scattering Problems
}

\begin{abstract}
The fast multipole method (FMM) was developed by Rokhlin to solve acoustic scattering problems very efficiently. We have modified and adapted it to the second-kind-integral-equation formulation of electromagnetic scattering problems in two dimensions. The present implementation treats the exterior Dirichlet (TM) problem for two-dimensional closed conducting objects of arbitrary geometry. The FMM reduces the operation count for solving the second-kind integral equation (SKIE) from $O\left(n^{3}\right)$ for Gaussian elimination to $O\left(n^{4 / 3}\right)$ per conjugated-gradient iteration, where $n$ is the number of sample points on the boundary of the scatterer. We also present a simple technique for accelerating convergence of the iterative method: "complexifying" $k$, the wavenumber. This has the effect of bounding the condition number of the discrete system; consequently, the operation count of the entire FMM (all iterations) becomes $O\left(n^{4 / 3}\right)$. We present computational results for moderate values of $k a$, where $a$ is the characteristic size of the scatterer.

\section{Comments}

Copyright 1992 IEEE. Reprinted from IEEE Transactions on Antennas and Propagation, Volume 40, Issue 6, June 1992, pages 634-641.

This material is posted here with permission of the IEEE. Such permission of the IEEE does not in any way imply IEEE endorsement of any of the University of Pennsylvania's products or services. Internal or personal use of this material is permitted. However, permission to reprint/republish this material for advertising or promotional purposes or for creating new collective works for resale or redistribution must be obtained from the IEEE by writing to pubs-permissions@ieee.org. By choosing to view this document, you agree to all provisions of the copyright laws protecting it.
\end{abstract}




\title{
The Fast Multipole Method (FMM) for Electromagnetic Scattering Problems
}

\author{
Nader Engheta, Senior Member, IEEE, William D. Murphy, Vladimir Rokhlin, and \\ Marius S. Vassiliou, Senior Member, IEEE
}

\begin{abstract}
The fast multipole method (FMM) was developed by Rokhlin to solve acoustic scattering problems very efficiently. We have modified and adapted it to the second-kind-integralequation formulation of electromagnetic scattering problems in two dimensions. The present implementation treats the exterior Dirichlet (TM) problem for two-dimensional closed conducting objects of arbitrary geometry. The FMM reduces the operation count for solving the second-kind integral equation (SKIE) from $O\left(n^{3}\right)$ for Gaussian elimination to $O\left(n^{4 / 3}\right)$ per conjugate-gradient iteration, where $n$ is the number of sample points on the boundary of the scatterer. We also present a simple technique for accelerating convergence of the iterative method: "complexifying"' $k$, the wavenumber. This has the effect of bounding the condition number of the discrete system; consequently, the operation count of the entire FMM (all iterations) becomes $O\left(n^{4 / 3}\right)$. We present computational results for moderate values of $k a$, where $a$ is the characteristic size of the scatterer.
\end{abstract}

\section{INTRODUCTION}

$E^{\prime}$ LECTROMAGNETIC scattering represents an important class of problems in physics and engineering. It is desirable to have efficient techniques to compute scattering accurately. In this paper we present the first application of Rokhlin's fast multipole method (FMM) to the problem of electromagnetic scattering from two-dimensional closed conducting objects of arbitrary geometry.

In many two-dimensional scattering problems, it is customary to reduce the scalar Helmholtz equation to a second-kind integral equation. The resulting integral equation can generally be treated using various numerical techniques [1], [2]. One of the standard methods for the numerical treatment of scattering problems is to discretize the second kind integral equation using an appropriate quadrature formula (Nyström's method) [1], [3]. Such discretization leads to systems of linear algebraic equations that may be solved by Gaussian elimination or iterative methods such as conjugate-gradient or generalized conjugate residual (GCR) [4]. These iterative

Manuscript received July 1, 1991; revised November 21, 1991. This work was supported by the Air Force Office of Scientific Research under Contract F49620-89-C-0048.

N. Engheta is with the Complex Media Laboratory, Moore School of Electrical Engineering, University of Pennsylvania, Philadelphia, PA 191046390.

W. D. Murphy and M. S. Vassiliou are with the Rockwell International Science Center, 1049 Camino Dos Rios, Thousand Oaks, CA 91360.

V. Rokhlin is with the Department of Computer Science, Yale University, New Haven, CT 06520 .

IEEE Log Number 9200431. methods require the full dense matrix to operate on a sequence of recursively generated vectors. Consequently, the operation count is $O\left(n^{2}\right)$ where $n$ is the dimension of the matrix. There have been many successful efforts to reduce the operation count and storage requirements and to introduce "fast" algorithms [5]-[8]. The FMM is a particularly promising method among these. In the FMM, the operation count for each iteration is reduced to $O\left(n^{4 / 3}\right)$, which is significantly smaller than $O\left(n^{2}\right)$, especially for large $n(>$ $10000)$. This algorithm can be further improved to one that has an operation count of $O(n \log n)$ per iteration. However, we have not yet implemented the fastest algorithm. When these algorithms are combined with a GCR or conjugategradient algorithm, the resulting procedure only requires a small number of iterations to converge to a solution of the scattering problem. This is the case even at resonance frequencies, if the method of "complexification" is applied (see discussion below). In addition, the $O\left(n^{4 / 3}\right)$ and $O(n \log n)$ algorithms are attained without any significant restriction on the shape of the scatterer other than that it be closed.

The purpose of the present paper is to explain this algorithm intuitively in simple terms and to explore its application to electromagnetic scattering. Rokhlin's FMM, which was first employed for Poisson's equation [5] and acoustic scattering [6] in two dimensions has been extended to three dimensions for Poisson's equation [9] and is currently being extended for Helmholtz's equation.

\section{Problem Statement}

Consider a two-dimensional conducting body whose axis is aligned with the $z$ coordinate axis. A monochromatic electromagnetic wave incident on this structure with an electric field vector parallel to the axis of the body is referred to as the transverse magnetic (TM) case. The incident and scattered fields both satisfy the following Helmholtz equation:

$$
\nabla^{2} E_{z}+k^{2} E_{z}=0
$$

where $k$ is the wavenumber equal to $2 \pi / \lambda$ with $\lambda$ the wavelength of the incident field in the exterior region. The boundary condition for (1) is that the total $\mathbf{E}$ field vanish on the surface of this conductor, i.e.,

$$
E_{z}^{\text {tot }}=0 \text { on } C
$$

or more explicitly

$$
E_{z}^{\text {inc }}+E_{z}^{\text {scat }}=0 \text { on } C .
$$


Here $C$ is the boundary of the scatterer. The above Helmholtz equation can be reduced to the following second-kind integral equation

$$
\Psi(\mathbf{r})+2 \int_{C} \frac{\partial G\left(k\left|\mathbf{r}-\mathbf{r}^{\prime}\right|\right)}{\partial n\left(\mathbf{r}^{\prime}\right)} \Psi\left(\mathbf{r}^{\prime}\right) d l^{\prime}=-2 E_{z}^{\text {inc }}(\mathbf{r})
$$

and the scattered field in the exterior region is computed by

$$
E_{z}^{\text {scat }}(\mathbf{r})=\int_{C} \frac{\partial G\left(k\left|\mathbf{r}-\mathbf{r}^{\prime}\right|\right)}{\partial n\left(\mathbf{r}^{\prime}\right)} \Psi\left(\mathbf{r}^{\prime}\right) d l^{\prime}
$$

where $\mathbf{r}$ and $\mathbf{r}^{\prime}$ are both on the boundary $C$, and $G$ is the free-space Green's function in two dimensions, i.e.,

$$
G\left(k\left|\mathbf{r}-\mathbf{r}^{\prime}\right|\right)=i H_{0}^{(1)}\left(k\left|\mathbf{r}-\mathbf{r}^{\prime}\right|\right) / 4
$$

with $H_{0}^{(1)}\left(k\left|\mathbf{r}-\mathbf{r}^{\prime}\right|\right)$ being the Hankel function of the first kind of order zero, where we are employing the $\exp (-i \omega t)$ time convention. See [10] for a derivation of (4). Equation (4), the TM case, is often referred to as the Dirichlet problem in the mathematical literature [1], [10]. If we discretize the boundary into $n$ points, then the above integral equation (4a) is converted to the following linear system (via Nyström's method [1]):

$$
\Psi\left(\mathbf{r}_{i}\right)+2 \sum_{j=1}^{n} A_{i j} \Psi\left(\mathbf{r}_{j}\right)=-2 E_{z}^{\mathrm{inc}}\left(\mathbf{r}_{i}\right)
$$

where the matrix $A=\left(A_{i j}\right)$ is $n \times n$ and the vectors $\left(\Psi\left(\mathbf{r}_{i}\right)\right)$ and $\left(E_{z}^{\mathrm{inc}}\left(\mathbf{r}_{i}\right)\right)$ are column vectors having $n$ rows. Applying normal matrix multiplication, $A \vec{\Psi}$ requires $O\left(n^{2}\right)$ operations. The FMM algorithm reduces this to $O\left(n^{4 / 3}\right)$ or ultimately to $O(n \log n)$.

Although most practitioners in electromagnetics are familiar with the method of moments for solving integral equations, fewer have used Nyström's method. The main difference is that in moment methods (or finite element techniques), the currents are expanded in a series of basis functions (pulse, hat, B-splines, etc.), and the unknowns are the weights multiplying these basis functions. In Nyström's method, the integrals are discretized using convergent quadrature formulas for a given kernel, and the unknowns are the current values at the sample points on the surface of the scatterer. Nyström's method has the advantage that it is easier to develop higher order quadrature formulas [3] than it is to employ higher order basis functions (as done in moment methods). In addition, matrix fill is more efficient with Nyström's method than with moment methods. See [1] and [3] for more details.

\section{Rapid Solution of Integral EQuations}

The detailed mathematics behind the FMM is presented in [5] and [6]. The development is quite complex. Below, we offer a simplified version, with more physical intuition relevant to electromagnetic scattering.

Consider $n$ nodes on the boundary of the scatterer. Divide the boundary into $p$ equal segments, where $2 \leq p<n$. In each segment, there are $n / p$ nodes. Fig. 1 illustrates a scatterer with $n$ nodes on its boundary. The figure shows the

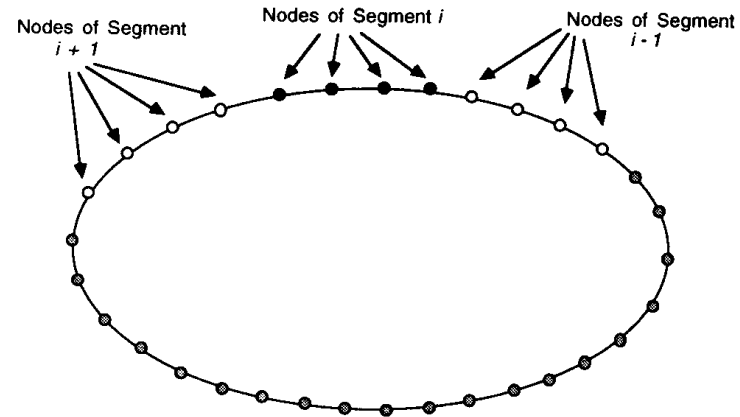

Fig. 1. Illustration of scatterer. Scatterer boundary is sampled by $n$ nodes equispaced in arc length. The nodes are grouped into $p$ segments, with each segment containing $n / p$ nodes. In this figure, $n=32$ and $p=8$, for the sake of illustration. The figure shows the nodes in the $i$ th segment (black) and its nearest-neighbor segments (white). Nodes shaded in grey belong to segments that are not near neighbors of segment $i$.

$i$ th segment and its two nearest neighbor segments. If the length of the boundary is $L$, each segment has length $L / p$. The center of each segment is located at $z_{i}(i=1,2, \cdots, p)$. In scattering problems, each node can be treated conceptually as if it were a source of radiation.

If we have sources within a finite region of space, the radiation emitted from these sources in the far zone can be approximated using a collection of multipoles located at the center of the region [5], [6], [11]. The multipole approximation converges rapidly outside any circle $D$ containing all sources and separated from $D$ by at least one wavelength. In fact, once a sufficiently large number of multipoles is included, the accuracy of the approximation increases superalgebraically (faster than any negative power of $N$, where $N$ is the number of multipoles employed) [6].

Consider each segment on the boundary as a cluster of $n / p$ sources. The sources in each segment are treated as a single aggregate source, and the radiation field of that equivalent source is approximated using the first $N$ multipoles located at the center of the segment. For each pair of sufficiently separated segments, the radiation of the $N$ multipoles of one segment can be represented as an analytical partial field expansion around the center of the other segment. Then from this information, the field at the other nodes of that segment can be evaluated using (16). For nearby segments, the direct contribution must be calculated to evaluate the radiation field. The radiation field at any particular node on the boundary is the sum of the contributions of $N$ multipoles of each of the far-away segments and the direct contribution of the nearby segments. The precise mathematical description of the process is considered in [6].

To illustrate the above verbal description mathematically, let us consider the scattered electric field in the exterior. In the TM case, according to (4b), the scattered field can be expressed in terms of a double-layer potential from a source on the boundary [10], which can be regarded as a fictitious source $\mathbf{K}(\vec{\rho})$, where $\vec{\rho}=(\rho, \theta)$, a point on $C$. For the 2-D case, this fictitious $\mathbf{K}$ has only a component in the $x y$ plane tangent to $C$, i.e., $\mathbf{K}(\vec{\rho})=K(\vec{\rho}) \hat{c}$, where $\hat{c}$ is a unit vector 
tangent to $C$. The current satisfying (4a) radiates the scattered field in the exterior region so that on the outside surface of $C, E_{z}^{\text {scat }}=-E_{z}^{\text {inc }}$. It must be noted that this current does not make the total electric field vanish in the interior region, which is not of interest here. The scattered electric field is given by

$$
\mathbf{E}^{\text {scat }}(\vec{\rho})=-\nabla \times \int_{C} G\left(k\left|\vec{\rho}-\vec{\rho}^{\prime}\right|\right) \mathbf{K}\left(\vec{\rho}^{\prime}\right) d l^{\prime} .
$$

In two dimensions, (7) can be written as

$$
E_{z}^{\text {scat }}(\vec{\rho})=\int_{C} \frac{\partial G\left(k\left|\vec{\rho}-\vec{\rho}^{\prime}\right|\right)}{\partial n\left(\vec{\rho}^{\prime}\right)} K\left(\vec{\rho}^{\prime}\right) d l^{\prime}
$$

which is identical to (4b) if $K(\vec{\rho})$ is taken to be $\Psi(\vec{\rho})$. Substituting (5) into (8), we get

$$
E_{z}^{\mathrm{scat}}(\vec{\rho})=(i / 4) \int_{C} \frac{\partial H_{0}^{(1)}\left(k\left|\vec{\rho}-\vec{\rho}^{\prime}\right|\right)}{\partial n\left(\vec{\rho}^{\prime}\right)} K\left(\vec{\rho}^{\prime}\right) d l^{\prime} .
$$

Consider a subsegment of $C$ defined as $C^{\prime}$. On this $C^{\prime}$ the Hankel function $H_{0}^{(1)}\left(k\left|\vec{\rho}-\vec{\rho}^{\prime}\right|\right)$ can be expanded in terms of higher order Hankel and Bessel functions:

$$
\begin{aligned}
& H_{0}^{(1)}(k|\vec{\rho}-\vec{\rho}|)=\sum_{m=-\infty}^{\infty} H_{m}^{(1)}(k \rho) J_{m}\left(k \rho^{\prime}\right) \\
& \cdot \exp \left(i m\left(\theta-\theta^{\prime}\right)\right)
\end{aligned}
$$

where $\rho^{\prime}<\rho$. The scattered electric field from $C^{\prime}$ can be written as

$$
\begin{aligned}
& E_{z}^{\text {scat }}(\rho, \theta)=\sum_{m=-\infty}^{\infty} H_{m}^{(1)}(k \rho) \exp (i m \theta) \\
& \cdot \int_{C^{\prime}}(i / 4) \frac{\partial J_{m}\left(k \rho^{\prime}\right) \exp \left(-i m \theta^{\prime}\right)}{\partial n\left(\rho^{\prime}, \theta^{\prime}\right)} K\left(\rho^{\prime}, \theta^{\prime}\right) d l^{\prime} .
\end{aligned}
$$

This can be regarded as one form of a multipole expansion of the source $\mathbf{K}\left(\rho^{\prime}, \theta^{\prime}\right)$. For a discretized source at $r$ points located at $x_{j}^{\prime}=\left(\rho_{j}^{\prime}, \theta_{j}^{\prime}\right)(j=1,2, \cdots, r)$ over $C^{\prime}$, (11) reduces to

$$
\begin{aligned}
E_{z}^{\mathrm{scat}}(\rho, \theta)=\sum_{m=-\infty}^{\infty} & \sum_{j=1}^{r}(i / 4) \frac{\partial J_{m}\left(k \rho_{j}^{\prime}\right) \exp \left(-i m \theta_{j}^{\prime}\right)}{\partial n\left(x_{j}^{\prime}\right)} \\
\cdot & H_{m}^{(1)}(k \rho) \exp (i m \theta) K\left(x_{j}^{\prime}\right) \Delta l_{j}^{\prime}
\end{aligned}
$$

where $\Delta l_{j}^{\prime}$ is the discretized element of arc length containing the source $K\left(x_{j}^{\prime}\right)$. For a given accuracy, we can truncate the infinite sum in (12) at $N$, and thus calculate the first $N$ multipoles of the source. This TM problem can be also formulated using an "equivalent" physical electric surface current.

The FMM algorithm was first applied to Poisson's equation for $n$ point charge lines at locations $x_{i}(i=1,2, \cdots, n)$ with strengths $\kappa_{i}$. This is mathematically equivalent to solving the equation

$$
\nabla^{2} \phi=\sum_{i=1}^{n} \delta\left(x-x_{i}\right) \kappa_{i}
$$

where $\delta(x)$ is the Dirac delta function and $x$ and $x_{i}$ are points in two dimensions. The solution to (13) is

$$
\phi(x)=\sum_{i=1}^{n} \kappa_{i} \log \left(\left|x-x_{i}\right|\right) /(2 \pi) .
$$

If we evaluate (14) at each point $x_{i}(i=1,2, \cdots, n)$, then this computation requires $O\left(n^{2}\right)$ operations. However, if large numbers of particles are combined into single computational elements, then this operation count can be reduced if an approximate answer (to a specified accuracy) is desired. When a cluster of particles is "far away" from a particular point, then the potential of the cluster is approximated by the potential induced by a single computational element located inside the cluster [8], [12]. In the FMM algorithm the computational element is a multipole expansion centered at a circle containing the cluster of particles. Given a cluster of charges located at points $z_{i}\left(i=1,2, \cdots, n_{c}\right)$, the expansion is given by

$$
\begin{aligned}
\phi(z) & =\operatorname{Re}\left(\sum_{i=1}^{n_{c}} \log \left(z-z_{i}\right)\right) \\
& \approx \operatorname{Re}\left(a_{0} \log \left(z-z_{0}\right)+\sum_{k=1}^{p} a_{k} /\left(z-z_{0}\right)^{k}\right) .
\end{aligned}
$$

Here $p$ is the order of the multipole and the $a_{k}$ are coefficients chosen so that the truncated series is an accurate approximation of the potential. The coefficients are determined from the Laurent expansion of $\log \left(z-z_{i}\right)$. For (15), the computational effort is only $O(p)$ operations and is much lower than $O\left(n_{c}\right)$ for the direct approach. The region must now be organized into well-separated points and very near points. For near points, the direct evaluation of (14) is used. See [5], [6], and [12] for details of decomposing the regions into boxes of different sizes. When applying the FMM to Helmholtz's equation instead of Poisson's equation the expansion (15) is replaced by the standard Hankel function expansion, which is then truncated to obtain a given accuracy requirement. That is,

$$
E_{z}^{\text {scat }}(\rho, \theta)=\left\{\begin{array}{l}
\sum_{m=-\infty}^{+\infty} \alpha_{m} H_{m}^{(1)}(k \rho) \exp (\operatorname{im} \theta), \\
\text { if } \rho>a \\
\sum_{m=-\infty}^{+\infty} \beta_{m} J_{m}(k \rho) \exp (\operatorname{im} \theta), \\
\text { if } \rho<a
\end{array}\right.
$$

depending on whether the calculations are to be done outside or inside the circle of radius $a$ [6]. Obviously, any prescribed accuracy in the series can be guaranteed by taking more terms in the expansion at the expense of more CPU time.

\section{Operation Count}

We illustrate intuitively the computational work required for the FMM algorithm using a simple example. For more details see [6]. Consider $p$ segments around the boundary of the scatterer. Assume that for each segment, the two adjacent segments are "nearby," and thus require direct calculation 
of the radiation field. All other segments are considered "far away" for this example and the multipole expansion can be used. The following steps are taken:

Step 1: Find the first $N$ multipoles of sources in each segment. Since to evaluate each multipole, all the sources are involved, the first $N$ multipoles of sources in each segment require $N n / p$ operations. For $p$ segments, we have

$$
N(n / p) p=N n \text {. }
$$

As mentioned earlier, the number of multipoles used is a function of the accuracy needed in the calculation. This number is typically proportional to $k d$ where $k=2 \pi / \lambda$ and $d$ is the length of the source region. Thus, $N \approx k L / p$. Substituting this value of $N$ into (17) gives

$$
N n \approx k(L / p) n
$$

as the operation count for this step.

Step 2: For each pair of "far-away" segments, evaluate the radiation fields of $N$ multipoles of one segment at the center of the other. This is an $O(N)$ operation for each pair. The number of pairs is almost $p^{2}$. In actuality, for each segment, the number of far-away segments is $p-3$. Therefore, the number of far-away pairs is $p(p-3)$, which for large $p$ is almost $p^{2}$. Therefore, the operation count for this step is

$$
N p^{2} \approx k(L / p) p^{2} \approx k L p .
$$

Step 3: In this step, for each segment, add the contribution of $N$ multipoles of any one of the far-away segments evaluated at the center of the chosen segment. For any chosen segment, the number of far-away segments is $p-3$, or approximately $p$, for large $p$. This step requires

$$
N p \approx k(L / p) p \approx k L
$$

operations for all $p$ segments.

Step 4: Here, the radiation field is known at the center of each segment. The field at the other nodes in the segment can be evaluated using a partial field expansion [6]. For each neighboring node, this is an $O(N)$ operation. Thus, for $n / p$ nodes in each segment, the number of operations is

$$
N n / p \text {. }
$$

For $p-3$ segments, we have

$$
(N n / p)(p-3) \approx N n \approx k(L / p) n .
$$

Step 5: Finally for the nearby (neighboring) segments, the direct contributions must be evaluated. For $q$ sources, the number of operations is $q^{2}$. Here, in each segment there are $n / p$ sources. For a particular segment in question and its two near-neighbors, the work is

$$
(3 n / p)^{2} \approx n^{2} / p^{2} \text {. }
$$

For $p$ segments, the count is

$$
\left(n^{2} / p^{2}\right) p \approx n^{2} / p
$$

Adding the above five steps and optimizing with respect to $p$ the resulting expression for total operation count (as in
[6]), the optimal-count $T$ is

$$
T=O\left(n^{3 / 2}\right)
$$

The operation count can be further reduced by applying the above procedure recursively, with each of the segments subdivided with appropriately chosen $p^{\prime}$. The new estimate so obtained shows that the FMM algorithm is $O\left(n^{4 / 3}\right)$. Our current FMM code implements the $O\left(n^{4 / 3}\right)$ algorithm. In [5] the above subdivision is used recursively to obtain an $O(n)$ algorithm when applied to Poisson's equation (13). By reproducing the construction of Section VII in [5] for Helmholt'z equation an $O(n \log n)$ algorithm is theoretically achievable.

The essential feature of the FMM algorithm is that it performs the matrix-vector operation

$$
A \vec{\Psi}^{(m)}(m=0,1,2, \cdots)
$$

in $O\left(n^{4 / 3}\right)$ operations. Here the superscript $m$ is the iteration counter. Note that $A$ is never computed, so that the algorithm only requires vector storage! The storage requirement is $O\left(n^{4 / 3}\right)$, as is the operation count [6]. The actual solution to the system (6) is obtained using a conjugate-gradient or GCR [4] algorithm in which the most computationally extensive step is that of forming the vector expression (26) by the FMM technique. GCR must be used on the matrix $I+2 A$ or conjugate-gradient on the normal equation because $I+2 A$ is nonsymmetric.

The FMM algorithm is in no way restricted to the TM case (4). In fact, an almost identical algorithm can be applied to the TE case (exterior Neumann problem in the mathematical literature [1], [10]) or to the combined field integral equation (CFIE), which does not have a resonance problem (see, e.g., [3]). Rather than solve the CFIE, we use a technique that employs the method of "complexification" on (4) directly. The "trick," the reader will soon see, works remarkably well and allows us to avoid dealing with the more complicated CFIE. Although "complexification" has been used before in electromagnetics to satisfy the radiation condition, here it is used for the purpose of reducing the condition number of the matrix $A$, thereby reducing the number of iterations for convergence of the conjugate-gradient algorithm.

\section{Computational Results}

In Fig. 2, we show the comparison of the FMM algorithm with the analytic solution for the scattering of a plane wave incident on a circular cylinder for the case $k a=80$. We present our computational results in a form often used in electrical engineering: that is, as plots of differential scattering cross section or radar cross section (RCS) versus observation angle, for a given angle of incidence. The RCS is related to the magnitude of the electric field in the far zone. In two dimensions, RCS is something of a misnomer: the more proper term is "scattering width" or "echo width," but the label RCS is commonly applied anyway. The two- 

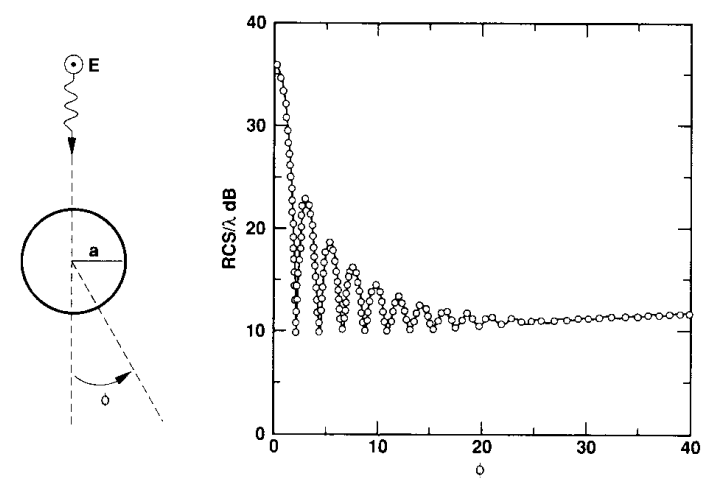

Fig. 2. RCS for a circular cylinder of unit radius. Incident field is a plane wave with wavenumber $k=80$. Solid line shows solution from the FMM code, while dotted line is that from the series solution.

dimensional definition is [13]

$$
\mathrm{RCS}=\sigma=2 \pi \lim _{r \rightarrow \infty} r \frac{\left|E_{z}^{\text {scat }}\right|^{2}}{\left|E_{z}^{\text {inc }}\right|^{2}}
$$

where $E_{z}^{\text {scat }}$ is the scattered field and $E_{z}^{\text {inc }}$ is the incident field. The quantity we plot is the ratio of $\sigma$ to the wavelength of the incident wave, expressed in decibels.

In Fig. 2 the agreement of the RCS between the two solutions is better than $0.001 \mathrm{~dB}$ for observation angles $\phi$ between $0^{\circ}$ and $40^{\circ}$, where most of the rapid changes occur. In this example, we have used 10 points per wavelength on the scatterer boundary, so $n=800$. In Table I, we list some results from the FMM code for various values of $k$ and $n$ for scattering from two-dimensional conducting circular and elliptical cylinders ( $a=$ semimajor axis, $b=$ semiminor axis). The expansions (16) were truncated to give an accuracy of $10^{-4}$ and the convergence tolerance for the conjugate gradient algorithm was set to $10^{-3}$. In the table, $N_{\text {iter }}$ denotes the actual number of iterations in the conjugate-gradient algorithm to achieve an error tolerance of $10^{-3}, \mathrm{ERR}=\| A \vec{\Psi}^{(m)}$ $-b \|_{2}$ (where $b$ here is the right-hand side of the linear system), and CPU is the average CPU time in seconds on a VAX 6410 computer for one iteration of the conjugate-gradient FMM algorithm using double precision arithmetic. In most cases, for "complexified" $k$-values an error tolerance of $10^{-4}$ instead of $10^{-3}$ would only add one or two more iterations to $N_{\text {iter }}$. However, for "noncomplexified" $k$-values, an error tolerance of $10^{-4}$ would require many more iterations because of the large condition number involved in those cases. Since the matrix $A$ is never explicitly calculated in the FMM algorithm, the total CPU time for one incident angle is roughly equal to the product of $N_{\text {iter }}$ and CPU. To calculate the number of points $n_{\lambda}$ per incident wavelength in Table I, we have used an approximate formula for the perimeter of an ellipse, obtaining

$$
n_{\lambda} \approx \frac{n}{k \sqrt{\left(a^{2}+b^{2}\right) / 2}} .
$$

Note that for some of the cases in Table I, we have used values of $k$, the wavenumber, that have imaginary parts.
TABLE I

Fast Multipole Results for Scattering from Two-Dimensional Conducting Circular and Elliptical Cylinders (DEC VAX 6410 COMPUTER, DOUBle Precision)

\begin{tabular}{lllllllc}
\hline$k b$ & $a$ & $b$ & $n$ & $n_{\lambda}$ & $N_{\text {iter }}$ & CPU & Err \\
\hline 50 & 1 & 1 & 500 & 10 & 66 & 11.99 & $0.441(-3)$ \\
80 & 1 & 1 & 800 & 10 & 79 & 22.35 & $0.856(-3)$ \\
$80+1.6 i$ & 1 & 1 & 800 & 10 & 13 & 22.43 & $0.200(-3)$ \\
$80+2.4 i$ & 1 & 1 & 800 & 10 & 9 & 21.73 & $0.655(-3)$ \\
$80+3.2 i$ & 1 & 1 & 800 & 10 & 8 & 21.89 & $0.198(-3)$ \\
$80+4.8 i$ & 1 & 1 & 800 & 10 & 6 & 21.27 & $0.750(-3)$ \\
$80+6.4 i$ & 1 & 1 & 800 & 10 & 6 & 21.22 & $0.223(-3)$ \\
$150+6.4 i$ & 1 & 1 & 1500 & 10 & 6 & 51.94 & $0.633(-3)$ \\
100 & 2 & 1 & 1600 & 10.12 & 184 & 34.89 & $0.992(-3)$ \\
$100+2 i$ & 2 & 1 & 1600 & 10.12 & 11 & 35.57 & $0.293(-3)$ \\
$100+3 i$ & 2 & 1 & 1600 & 10.12 & 9 & 36.53 & $0.478(-3)$ \\
$100+4 i$ & 2 & 1 & 1600 & 10.12 & 8 & 35.84 & $0.494(-3)$ \\
$100+6 i$ & 2 & 1 & 1600 & 10.12 & 6 & 35.35 & $0.998(-3)$ \\
50 & 3 & 1 & 1200 & 10.73 & 95 & 32.61 & $0.950(-3)$ \\
$50+2 i$ & 3 & 1 & 1200 & 10.73 & 10 & 33.98 & $0.863(-3)$ \\
$100+2 i$ & 3 & 1 & 2237 & 10 & 12 & 86.93 & $0.639(-3)$ \\
\hline
\end{tabular}

$k=$ wavenumber.

$a=$ semimajor axis.

$b=$ semiminor axis.

$n=$ total number of unknowns (sample points on scatterer boundary).

$n_{\lambda}=$ number of unknowns per wavelength of incident radiation.

$N_{\text {iter }}=$ number of iterations to convergence.

$\mathrm{CPU}=\mathrm{CPU}$ time per iteration, in seconds.

$\mathrm{Err}=$ relative error

Notation: $0.441(-3)$ means $0.441 \times 10^{-3}$.

This was done to test the method of "complexification." Equation (4) has resonances [10] only at real distinct values of $k$ and as $k$ increases, root clusters become more dense; at these resonance frequencies, the condition number of the matrix $A$ becomes large and iterative methods require many more iterations to converge to a given tolerance. We have shown in [3] that by moving $k$ slightly into the complex plane ("complexifying" $k$ ) the condition number can be reduced by four or five orders of magnitude and consequently, the convergence rate of most iterative methods can be greatly improved. This is clearly demonstrated in Table I. Furthermore, by using two complex values of $k$ (say $k=$ $100+0.1 i$ and $k=100+0.2 i$ ) we have shown in [3] that the extrapolated values of the RCS to the real axis are accurate to a value in decibels roughly equal to the amount of movement into the complex plane (in this case $0.1 \mathrm{~dB}$ ). Of course, parabolic extrapolation would be even more accurate, but would require computations for three complex values of $k$. However, this is not a severe problem when using iterative methods because the iteration scheme for the second value of $k$ can be started with the final solution from the first value of $k$ and so on. Thus two solutions for two close values of $k$ require less than two times as much CPU time as does one case. Complexification is hence a viable and relatively inexpensive method for accelerating convergence in such problems.

Parabolic extrapolation is done in the following way. Suppose the imaginary parts of three "complexified" $k$-values are $k_{0}, k_{1}$, and $k_{2}$ and the corresponding RCS values for one observation angle are $\sigma_{0}, \sigma_{1}$, and $\sigma_{2}$, respectively. We compute the Lagrange interpolation polynomial through the points $\left(k_{0}, \sigma_{0}\right),\left(k_{1}, \sigma_{1}\right)$ and $\left(k_{2}, \sigma_{2}\right)$ and set the imagi- 
nary $k$ equal to zero in this quadratic polynomial in $k$ (i.e., we extrapolate to the real axis) obtaining

$$
\sigma(0)=p_{0} \sigma_{0}+p_{1} \sigma_{1}+p_{2} \sigma_{2}
$$

where $p_{0}=k_{1} k_{2} /\left[\left(k_{0}-k_{1}\right)\left(k_{0}-k_{2}\right)\right], p_{1}=k_{0} k_{2} /\left[\left(k_{1}\right.\right.$ $\left.\left.-k_{0}\right)\left(k_{1}-k_{2}\right)\right]$, and $p_{2}=k_{0} k_{1} /\left[\left(k_{2}-k_{0}\right)\left(k_{2}-k_{1}\right)\right]$. Parabolic extrapolation is second-order accurate provided the function $\sigma(k)$ is "sufficiently smooth" (has three continuous derivatives). This means that for uniform spacing ( $\Delta k=k_{2}$ $-k_{1}=k_{1}-k_{0}$ ) the error in $\sigma$ due to the extrapolation is $O\left((\Delta k)^{2}\right)$. Linear extrapolation is only $O(\Delta k)$ if $\sigma$ has two continuous derivatives in $k$. In practice, we have found that the accuracy of the extrapolation procedure is even better than these estimates would suggest.

In Fig. 3, we show some RCS plots for scattering from a two-dimensional conducting circular cylinder for linear extrapolation from "complexified" $k$-values. To remove the effect of the "complexification" in computing the RCS in the far field, we must rescale $E_{z}^{\text {scatt }}\left(r_{\mathrm{FF}}\right)$ where $r_{\mathrm{FF}}$ is the value of $r$ in the far field, typically about 10000 . If $k=k_{\text {re }}+i k_{\text {im }}$ where $k_{\mathrm{im}}$ is the imaginary term added to $k$ to accelerate convergence of the iterative procedure, then we rescale $E_{z}^{\text {scat }}\left(r_{\mathrm{FF}}\right)$ by multiplying it by $\exp \left(k_{\mathrm{im}} r_{\mathrm{FF}}\right)$ from the asymptotic expansion of $H_{v}^{(1)}\left(k_{\mathrm{im}} r_{\mathrm{FF}}\right)$. The RCS is then computed using (27) and the rescaled values of $E_{z}^{\mathrm{scat}}\left(r_{\mathrm{FF}}\right)$. Extrapolation of the RCS to the real axis is also done using the rescaled values. In Fig. 3, the linearly extrapolated values (from $k=80+0.5 i$ and $k=80+1.0 i$ ) of the RCS give agreement to $0.001 \mathrm{~dB}$ with the unextrapolated ones (for $k=80$ ). The parabolically extrapolated values give the same excellent agreement. The number of iterations required to converge to an error tolerance of $10^{-3}$ for these three cases are 79,26 , and 16 , respectively, and again we see that for faster convergence it pays to "complexify." Note also that large condition numbers not only imply slow convergence but also poor accuracy, giving another reason to "complexify." Of course, one could always solve the CFIE rather than (4) and avoid the problem of large condition numbers due to resonance. Unfortunately, solving the CFIE for TE polarization requires dealing with the second derivative of the free space Green's function, which has a difficult singularity. "Complexification" is easier!

In Fig. 4, we plot the RCS for scattering from a conducting elliptical cylinder using linear extrapolation ( $a=2, b=$ $1, k=100+0.5 i, k=100+1.0 i$ ) with a plane wave incident at $90^{\circ}$. As can be seen in Table I, $n=1600$ for these cases. The agreement between extrapolated values and unextrapolated values of the RCS is better than $.001 \mathrm{~dB}$. We only show the extrapolated values in the figure, to avoid too much congestion. Parabolic extrapolation using $k=100+$ $0.3 i, 100+0.6 i, 100+0.9 i$ gives the same excellent results.

As we have stated, the FMM algorithm of this paper is $O\left(n^{4 / 3}\right)$ per conjugate-gradient iteration. This means that the CPU time for a given scatterer is proportional to $n^{4 / 3}$, i.e.,

$$
T=\alpha_{\mathrm{VAX}} n^{4 / 3}
$$

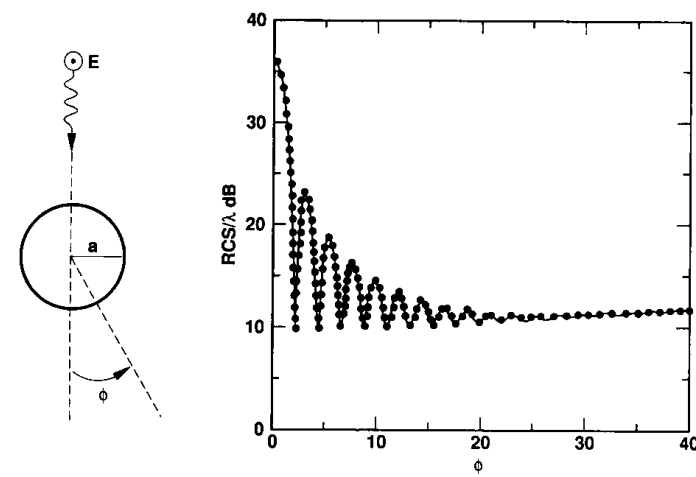

Fig. 3. RCS for a circular cylinder of unit radius. Incident fields are plane waves with wavenumbers $k=80, k=80+0.5 i$, and $k=80+1.0 i$. Solid line shows solution from the FMM code with no extrapolation ( $k=$ 80 ). Dotted line shows solution of the linearly extrapolated RCS from $k=80+0.5 i$ and $k=80+1.0 i$.
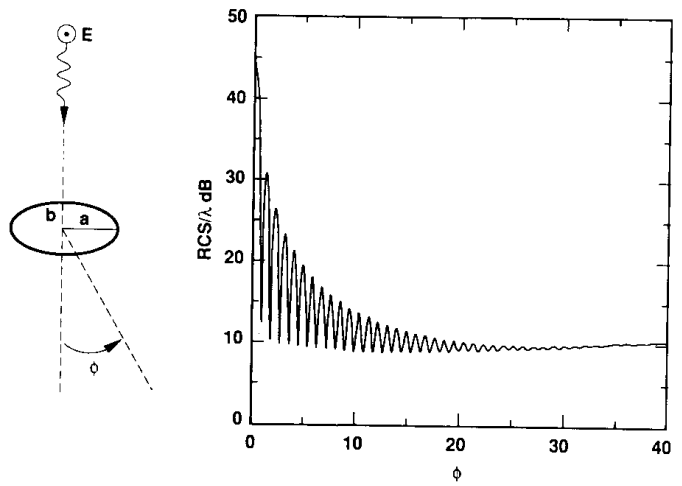

Fig. 4. RCS results from the FMM code for an elliptical cylinder with semimajor axis $a=2$ and semiminor axis $b=1$ linearly extrapolated from solutions for incident field plane waves at $90^{\circ}$ with wavenumbers $k=100$ $+0.5 i$ and $k=100+1.0 i$.

where we have emphasized that the proportionality constant $\alpha$ is a function of the computer being used (in this case a VAX 6410 in double precision). Using (30) and some of the data in Table I, we can obtain a rough estimate for the value of $\alpha_{\mathrm{VAX}}$. In this case

$$
\alpha_{\mathrm{VAX}} \approx 0.003
$$

Suppose now we would like to estimate the CPU time required on our VAX to compute the electromagnetic scattering problem for an example having 10000 unknowns. We assume that with some reasonable level of "complexification," convergence occurs in 10 iterations or less. Thus, for this example

$$
T^{\mathrm{tot}} \approx \alpha_{\mathrm{VAX}}(10) n^{4 / 3}=0.03(10000)^{4 / 3}=1.8 \mathrm{~h} .
$$

Although this last CPU time may seem large, we are only using a VAX in double precision, and a vectorized version of this code on a CRAY computer would do the same calculation in minutes. Furthermore, ordinary Gaussian elimination for a matrix of order 10000 would require about $(10000)^{3} / 3$ 
operations and would use considerable more CPU time than that in (32).

For $m$ incident fields, Gaussian elimination would require $O\left(n^{3}+m n^{2}\right)$ operations, and the FMM would have to be repeated $m$ times requiring $O\left(m n^{4 / 3}\right)$ operations. If $m \ll n$, clearly the FMM is superior. However, even in the very worst case when $m=O(n)$, the FMM beats Gaussian elimination because $O\left(n^{7 / 3}\right)$ is better than $O\left(n^{3}\right)$. Furthermore, in either case for $m$ incident fields Gaussian elimination requires $O\left(n^{2}\right)$ storage allocations while the FMM only requires $O\left(n^{4 / 3}\right)$, and in many large problems, storage requirements on a given computer may be a more limiting factor than CPU requirements.

Finally, the most important statement about the FMM and our approach to "complexification" and extrapolation of the $\mathrm{RCS}$ values for complex $k$-values to the real axis is that for complex $k(\operatorname{Re}(k)>0$ and $\operatorname{Im}(k)>0)$, the solution to (4), a second-kind integral equation, is unique for sufficiently smooth scatterers, and consequently, the condition number of $A$ is asymptotically bounded and independent of $n$. A proof of this result is given in [14]. This means, when we use our extrapolation procedure (either linear or parabolic), $N_{\text {iter }}$ is small (say, $N_{\text {iter }} \leq 10$ ) and independent of $n$ for error tolerances of $10^{-3}$. Of course, if the accuracy requirements are increased, so will $N_{\text {iter }}$, but in either case the FMM is globally $O\left(n^{4 / 3}\right)$ and we can write

$$
T^{\text {tot }}=N_{\text {iter }} \alpha_{\text {computer }} n^{4 / 3}
$$

where $N_{\text {iter }}$ is only a function of $\epsilon$, the error tolerance, and not a function of $n . \alpha_{\text {computer }}$ is a function of the computer speed for arithmetic operations, and is also independent of $n$. We present more evidence for this in Table II, where we have set the "complexification" to $5 i$ in all cases and maintained approximately 10 points per wavelength for various geometries. Note the small number of iterations to convergence, indicating a bounded condition number independent of $n$. Finally, in Table III, we list some preliminary results of the FMM code on the CRAY-2. This code is not yet fully vectorized, so we do not give CPU times.

Although we have only considered circular and elliptical cylinders for the test cases in this paper, our code can handle any closed two-dimensional metal scatterer having a unique outward normal at every point on $C$. Therefore, at present, we cannot deal with the trailing edge of an airfoil that comes to a point (nonexistence of $\partial G / \partial n$ at the trailing edge); currently, we must round out this region slightly. However, replacing the current at this singular point with its asymptotic expansion will allow us to alleviate this difficulty. We are also considering thin coatings about metal scatterers using higher order impedance boundary conditions, similar to our treatment in [15].

For more examples of the FMM applied to more complicated scatterers, and comparisons of the method with other techniques, we refer the reader to [16], which considers a variety of scattering problems. It explores the issue of how much "complexification" can be applied without unduly sacrificing accuracy in the solution. As a rough "rule of
TABLE II

Fast Multipole Method: CPU Time for Fixed "Complexification" (Scattering from Two-Dimensional Conducting Circular and Elliptical Cylinders) (DEC VAX 6410 COMPUTER, Double Precision)

\begin{tabular}{llllllc}
\hline$k b$ & $a$ & $b$ & $n$ & $N_{\text {iter }}$ & CPU & Err \\
\hline $50+5 i$ & 1 & 1 & 500 & 6 & 11.97 & $0.251(-3)$ \\
$100+5 i$ & 1 & 1 & 1000 & 6 & 22.46 & $0.973(-3)$ \\
$150+5 i$ & 1 & 1 & 1500 & 7 & 42.28 & $0.313(-3)$ \\
$200+5 i$ & 1 & 1 & 2000 & 7 & 51.90 & $0.686(-3)$ \\
$50+5 i$ & 2 & 1 & 791 & 7 & 22.22 & $0.998(-3)$ \\
$150+5 i$ & 2 & 1 & 2372 & 8 & 78.59 & $0.408(-3)$ \\
$100+5 i$ & 3 & 1 & 2237 & 9 & 86.28 & $0.999(-3)$ \\
\hline
\end{tabular}

$k=$ wavenumber

$a=$ semimajor axis.

$b=$ semiminor axis.

$n=$ total number of unknowns (sample points on scatterer boundary).

$N_{\text {iter }}=$ number of iterations to convergence.

$\mathrm{CPU}=\mathrm{CPU}$ time per iteration, in seconds.

$\mathrm{Err}=$ relative error .

Notation: $0.251(-3)$ means $0.251 \times 10^{-3}$

TABLE III

Fast Multipole Method: CRAY Results with Fixed "COMPLEXIFICATION" (SCATTERING FROM TWODimensional Conducting Circular and Elliptical Cylinders) (CRAY-2 COMPUTER, Single Precision)

\begin{tabular}{llllcc}
\hline$k b$ & $a$ & $b$ & $n$ & $N_{\text {iter }}$ & Err \\
\hline $200+5 i$ & 1 & 1 & 2000 & 7 & $0.686(-3)$ \\
$300+5 i$ & 1 & 1 & 3000 & 8 & $0.331(-3)$ \\
$400+5 i$ & 1 & 1 & 4000 & 8 & $0.803(-3)$ \\
$500+5 i$ & 1 & 1 & 5000 & 9 & $0.327(-3)$ \\
$100+5 i$ & 2 & 1 & 1582 & 7 & $0.997(-3)$ \\
$200+5 i$ & 2 & 1 & 3163 & 6 & $0.995(-3)$ \\
$300+5 i$ & 2 & 1 & 4744 & 6 & $0.991(-3)$ \\
$100+5 i$ & 3 & 1 & 2237 & 8 & $0.995(-3)$ \\
$200+5 i$ & 3 & 1 & 4473 & 7 & $0.997(-3)$ \\
$100+5 i$ & 4 & 1 & 2916 & 9 & $0.998(-3)$ \\
$50+5 i$ & 10 & 1 & 3554 & 8 & $0.996(-3)$ \\
\hline
\end{tabular}

$k=$ wavenumber

$a=$ semimajor axis

$b=$ semiminor axis.

$n=$ total number of unknowns (sample points on scatter boundary).

$N_{\text {iter }}=$ number of iterations to convergence

Err $=$ relative error

Notation: $0.686(-3)$ means $0.686 \times 10^{-3}$.

thumb,"' complexification at $1 \%$ of $k a$ works well for many problems. The readers should also note that the method of complexification and extrapolation stands on firm mathematical ground. The book by Leis [17] provides a comprehensive discussion of the principle of limiting absorption, which forms the mathematical basis for the method.

Our closing observation is that in computational electromagnetic scattering, reasonable results can usually be obtained if $k a$ is small. It is much more difficult to get good results for large values of $k a$, and it is doubly difficult to do so efficiently. We hope that by choosing moderate values of $k a$, we have demonstrated the robustness of the FMM algorithm for effectively solving electromagnetic scattering problems.

\section{REFERENCES}

[1] R. Kress, Linear Integral Equations. Berlin: Springer-Verlag, 1989.

[2] R. F. Harrington, Field Computation by Moment Methods. Malabar, FL: Krieger, 1968 
[3] W. D. Murphy, V. Rokhlin, and M. S. Vassiliou, "Solving electromagnetic scattering problems at resonance frequencies," J. Appl. Phys., vol. 67, pp. 6061-6065, May 15, 1990.

[4] S. C. Eisenstat, H. C. Elman, and M. H. Schultz, "Variational iterative methods for nonsymmetric systems of linear equations," SIAM J. Numer. Anal., vol. 20, pp. 345-357, Apr. 1983.

[5] V. Rokhlin, "Rapid solution of integral equations of classical potential theory," J. Comput. Phys., vol. 60, pp. 187-207, Sept. 15, 1985.

[6] -, "Rapid solution of integral equations of scattering theory in two dimensions," J. Comput. Phys., vol. 86, pp. 414-439, Feb. 1990

[7] F. X. Canning, "The impedance matrix localization (IML) method for moment-method calculations,"' IEEE Antennas Propagat. Mag., vol. 32, pp. 18-30, May 1990.

[8] C. R. Anderson, "An implementation of the fast multipole method without multipoles," UCLA Computational and Applied Mathematics CAM Rep. 90-14. Los Angeles: UCLA, July 1990.

[9] L. Greengard and V. Rokhlin, "The rapid evaluation of potential fields in three dimensions," Lecture Notes in Mathematics 1360, C. Anderson and L. Greengard, Eds. Berlin: Springer-Verlag, 1988, pp. 121-141.

[10] D. Colton and R. Kress, Integral Equation Methods in Scattering Theory. New York: Wiley, 1983.

[11] C. H. Papas, Theory of Electromagnetic Wave Propagation. New York: Dover, 1988.

[12] L. Greengard, The Rapid Evaluation of Potential Fields in Particle Systems, Yale Univ., New Haven, CT: Yale University, Dept. Comput. Sci. Res. Rep. YALEU/DCS/RR-553, Apr. 1987.

[13] G. Ruck, D. E. Barrick, W. D. Stuart, and C. K. Krichbaum, Radar Cross Section Handbook, vol. 1. New York: Plenum, 1970.

[14] K. E. Atkinson, A Survey of Numerical Methods for the Solution of Fredholm Integral Equations of the Second Kind. Philadelphia, PA: SIAM, 1976.

[15] B. Engquist, W. D. Murphy, and M. S. Vassiliou, "Higher order impedance boundary conditions," J. Appl. Phys., to be submitted.

[16] W. D. Murphy, V. Rokhlin, and M. S. Vassiliou, "Acceleration methods for the iterative solution of electromagnetic scattering problems," Radio Sci., submitted.

[17] R. Leis, Initial Boundary Value Problems in Mathematical Physics. New York: Wiley, 1986.

Nader Engheta (S'80-M'82-SM'89), for a photograph and biography please see page 373 of the April 1992 issue of this Transactions.

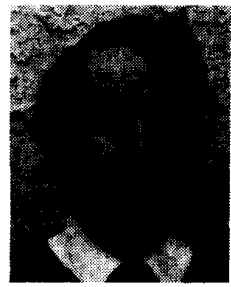

William D. Murphy received the B.S. degree in chemistry from the University of California, Berkeley, in 1961 and the Ph.D. degree in numerical analysis from the Courant Institute of Mathematical Sciences, New York University, New York, in 1966.

He has been employed at Rockwell International, Thousand Oaks, CA, since 1966 where he is a member of the Technical Staff in the Computational Sciences Function. He has the corporate responsibility of determining Rockwell's future needs in mathematical software and maintaining large software libraries on three separate computing systems (IBM, CDC, and CRAY). For the last several years he has worked in the areas of transonic flow, laminar and turbulent three-dimensional flow for wall jets and jet flaps, multigrid methods for various nonlinear partial differential equations, FMM for electromagnetic scattering problems, and process and device modeling for semiconductors. He has much part-time teaching experience at the University of California (Berkeley, Irvine, Los Angeles), New York University, University of London (one-year leave of absence), and California State University (Northridge, Fullerton). His main interest has been in developing large application codes in the field of laser optics, electromagnetics, VLSI, fluid dynamics, and chemical physics. He is the author of more than 70 scientific papers.

Vladimir Rokhlin received the M.S. degree in mathematics from Vilnius University, Lithuania, in 1973, and the Ph.D. degree in applied mathematics from Rice University, Houston, TX, in 1983.

He was an instructor at Vilnius University from 1972-1973 and served as a mathematician at the Institute of Arctic Geology in Leningrad from 1973-1976. In 1976 he immigrated to the U.S. and worked as a consultant at Computer Systems, Houston, TX, until 1978. Froni 1978-1979 he was a partner in Livshitz and Associates, Houston, TX, and in 1979 he joined Exxon Production Research Company, Houston, TX, as a Senior Research Specialist. In 1985 he joined Yale University, New Haven, CT. He has authored 30 papers in applied mathematics, numerical analysis, and computational science. He has contributed significantly to the numerical solution of integral equations arising in scattering theory.

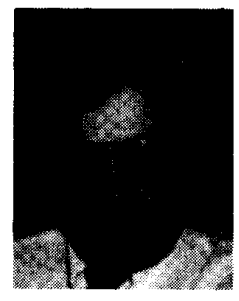

Marius S. Vassiliou (S'81-M'83-SM'90) received the bachelor's degree (with highest honors) from Harvard University, Cambridge, MA, the M.S. degree in computer science from the University of Southern California, Los Angeles, the M.S. degree from the California Institute of Technology, Pasadena, the M.B.A. degree from the University of California, Los Angeles, and the Ph.D. degree in geophysics and electrical engineering from the California Institute of Technology in 1983.

From 1983-1985 he was a Senior Research Scientist at ARCO Oil and Gas Company's Research and Development Laboratories, where he developed advanced software for seismic interpretation and analysis. He joined the Rockwell International Science Center, Thousand Oaks, CA, in 1985. He is a member of the Technical Staff in the Computational Sciences Function. He has worked in geophysics, ultrasonics, materials science, and electromagnetics, mostly from the computational point of view. He has also done significant work on computational analysis for the printing industry. He has authored 25 publications in technical journal and books, and a like number of internal research reports. He has taught at the University of Texas, Dallas, and at Moorpark College.

Dr. Vassiliou is a member of ACM, SEG, and Sigma Xi. 